\title{
Titan's gravity field and interior structure after Cassini
}

\author{
Daniele Durante $^{\mathrm{a}, *}$, D.J. Hemingway ${ }^{\mathrm{b}, \mathrm{c}}$, P. Racioppa ${ }^{\mathrm{a}}$, L. Iess ${ }^{\mathrm{a}}$, D.J. Stevenson ${ }^{\mathrm{d}}$ \\ ${ }^{a}$ Department of Mechanical and Aerospace Engineering, Sapienza University of Rome, Italy \\ ${ }^{\mathrm{b}}$ Department of Earth and Planetary Science, University of California, Berkeley, CA, USA \\ ${ }^{\mathrm{c}}$ Department of Terrestrial Magnetism, Carnegie Institution for Science, Washington, DC, USA \\ ${ }^{\mathrm{d}}$ Division of Geological and Planetary Sciences, California Institute of Technology, Pasadena, CA, USA
}

\section{A R T I C L E IN F O}

\section{Keywords:}

Geophysics

Orbit determination

Radioscience

Titan interior

\begin{abstract}
A B S T R A C T
Since its arrival at Saturn in 2004, Cassini performed nine flybys devoted to the determination of Titan's gravity field and its tidal variations. Here we present an updated gravity solution based on the final data set collected during the gravity-dedicated passes, before Cassini's plunge into Saturn's atmosphere. The data set includes an additional flyby (T110, March 2015, primarily devoted to imaging Titan's north polar lakes) carried out with the low-gain antenna. This flyby was particularly valuable because the closest approach occurred at a high latitude $\left(75^{\circ} \mathrm{N}\right)$, over an area not previously sampled.

Previously published gravity results (Iess et al., 2012) indicated that Titan is subject to large eccentricity tides in response to the time varying perturbing potential exerted by Saturn. The magnitude of the response quadrupole field, expressed in the tidal Love number $k_{2}$, was used to infer the existence of an internal ocean. The new gravity field determination provides an improved estimate of $k_{2}$ of about 0.62 , accurate to a level of a few percent. The value is higher than the simplest models of Titan suggest and the interpretation is unclear; possibilities include a high density ocean (as high as $1300 \mathrm{~kg} / \mathrm{m}^{3}$ ), a partially viscous response of the deeper region, or a dynamic contribution to the tidal response. The new solution includes higher degree and order harmonic coefficients (up to 5) and offers an improved map of gravity anomalies. The geoid is poorly correlated with the topography, implying strong compensation. In addition, the updated geoid and its associated uncertainty could be used to refine the gravity-altimetry correlation analysis and for improved interpretation of radar altimetric data.
\end{abstract}

\section{Introduction}

On 15 September 2017, Cassini plunged into Saturn's atmosphere and completed its long-lasting mission. After 7 years spent in deep space and 13 years orbiting Saturn, the spacecraft collected an incredible amount of scientific data about the Saturnian system. One of the main objectives of the mission was the study of Titan, Saturn's largest moon. Also, Titan was crucial for navigating Cassini around the system because it was exploited to perform large orbital correction maneuvers with low propellant cost. For this reason, Cassini performed 124 flybys of Titan during the mission (labelled with a T, which stands for Titan, followed by the number of the pass, e.g., T011), nine of which were entirely dedicated to the study of its gravity field, with the aim of inferring its interior structure. During the gravity-dedicated Titan passes, Cassini pointed its High-Gain Antenna (HGA) toward Earth, ensuring the best possible data quality. Since Cassini lacks a scan platform, this attitude does not simultaneously allow the operation of the other instruments that require pointing. Also, during gravity passes, thruster firings, commonly used for attitude maneuvers, were absent in order to preserve the coherency of Cassini's orbit, allowing an accurate reconstruction of the dynamical system. In addition to the nine gravitydedicated Titan passes, during the high-altitude T110 pass the gravity data were acquired using the omnidirectional Low-Gain Antenna (LGA) while Cassini's Visual and Infrared Mapping Spectrometer (VIMS) took a high-resolution regional map of Titan's north polar lakes.

The analysis of the first four gravity flybys (Iess et al., 2010) reported accurate measurements of Titan's gravity field up to degree 3 . The inclusion of two additional flybys (Iess et al., 2012) shed light on Titan's tidal response to eccentricity tides, revealing its large deformability over timescales of the orbital period, an indication of a global ocean at depth. Here, we update Titan's gravity field and its internal structure by including the data collected during three additional gravity-dedicated flybys and the T110 pass.

The main details of the passes when Cassini collected gravity data

\footnotetext{
* Corresponding author.

E-mail address: daniele.durante@uniroma1.it (D. Durante).
} 
are reported in Table 1. Each pass has a different orbital geometry, but the spatial coverage of Titan is not uniform (see Fig. 1a); the equatorial region of the planet has been sampled more than the polar regions. To determine Titan's tidal response to Saturn at its orbital frequency, the mean anomaly is the key quantity (Iess et al., 2012). Cassini's sampling of Titan's mean anomalies is very broad (see Fig. 1b) and allows us to further constrain Titan's tidal behavior. The Sun-Earth-Probe (SEP) angle is very important in determining the Doppler noise in the radio link, due to the effect of solar plasma noise turbulence (Asmar et al., 2005; Iess et al., 2014). The Doppler noise budget is influenced also by a daily variability due to the troposphere conditions at the ground station. For these reasons, the Doppler noise varies from a minimum of $0.017 \mathrm{~mm} / \mathrm{s}$ during T011 to a maximum of $0.068 \mathrm{~mm} / \mathrm{s}$ during T045 (see Table 1).

\section{Data analysis}

Titan's updated gravity field exploits the full data set, which includes mostly two-way Doppler data. After Cassini's Ka-band translator failed in 2003, two- or three-way Doppler data are available only with $\mathrm{X}$-band uplink $(7.2 \mathrm{GHz}$ ) and $\mathrm{X}$ or Ka-band downlink (respectively 8.4 and $32.0 \mathrm{GHz}$ ). The $\mathrm{X}$-band uplink makes the radio link especially sensitive to charged particle effects, which increase dramatically when the SEP angle is small (i.e., near solar conjunctions; see Asmar et al., 2005; Iess et al., 2014). In the case where multiple data types are available in the same time interval, the data selection privileges twoway Doppler data over three-way data, and Ka-band over X-band in downlink. The three-way data are about $12 \%$ of the total Doppler points. We discarded data acquired when the elevation angle of the spacecraft, as viewed from the ground station, is below $20^{\circ}$ to prevent bias in the solution that may come from uncalibrated troposphere. We weighted the data from each tracking pass according to the observed noise RMS. Each observation arc spans about 2 to 5 days about the time of Cassini's closest approach (C/A) to Titan, and avoids orbit correction maneuvers and thruster firing (used to desaturate Cassini's reaction wheels). Range data have not been included because they do not contribute substantially to the determination of Titan's gravity field; also, since range data are more affected by systematic errors, we prefer a solution with Doppler data only. However, range data have been used afterward to test the robustness of the solution based on Doppler data only. While, as expected, range data do not change the estimated gravity coefficients, they are used to update the ephemerides of Saturn in the solar system.

The Doppler data have been compressed at $60 \mathrm{~s}$ and analyzed with MONTE, the JPL orbit determination code (Evans et al., 2018). Cassini's dynamical model includes the gravitational effect in a relativistic context due to the Solar System planets including Saturn and its satellites. For Saturn, the zonal gravity field up to degree 10 has been included, in agreement with the dynamical model used for Titan's ephemerides (see Jacobson et al., 2006 and subsequent JPL releases). Titan's gravity field coefficients are included up to degree and order 5 . The rotational elements have been adopted from Meriggiola et al. (2016), obtained from the analysis and georeferencing of Cassini RADAR images. We included non-gravitational accelerations from the solar radiation pressure and the anisotropic thermal emission produced by the three onboard Radioisotope Thermoelectric Generators (RTGs). In addition, low-altitude flybys (in particular T022 and T068) require the inclusion of an exponential model for the density of Titan's upper atmosphere since the drag acceleration produced at those altitudes is large enough to perturb Cassini's trajectory in a non-negligible way (the peak acceleration in T022 is of the order of $3 \times 10^{-6} \mathrm{~m} / \mathrm{s}^{2}$ ).

The Doppler data from the different passes are collected in a multiarc, least-squares estimation filter, which provides the corrections to the dynamical model's parameters and, after a few iterations, allows us to fit the Doppler data to the noise level. In each arc, we solve for both Cassini's and Titan's state vectors at the beginning of each observation arc. The initial values for Cassini's state vectors are taken from the latest release of the navigation team for each pass, with a priori uncertainties on position and velocity of, respectively, $2 \mathrm{~km}$ and $2 \mathrm{~cm} / \mathrm{s}$ in all the direction, large enough not to constrain the solution. Particular attention should be paid to Titan's state vectors and the details are discussed below. We combine the information from all the arcs to solve for Titan's gravity field coefficients up to degree and order 5, its gravitational parameter, the tidal Love number $k_{2}$, the density of Titan's atmosphere at a reference altitude and its scale height, and the spacecraft bodyfixed acceleration from the onboard RTGs. All these quantities have a priori uncertainties which do not constrain the estimate. The solar radiation pressure acceleration is not estimated but considered ${ }^{1}$ with an uncertainty of $5 \%$. The uncertainty on Titan's rotation model from SAR imaging (Meriggiola et al., 2016) is much lower than that achievable with gravity measurements and thus the rotational parameters have not been estimated.

The analysis of T110 involves one additional challenge, related to the use of Cassini's LGA. Since the main purpose of this Titan flyby was to take a high-resolution regional map of Titan's north polar lakes with Cassini's VIMS, the HGA was not pointed toward the Earth, and the phase-coherent radio link was established through the LGA. As the spacecraft attitude changes over time, the phase center of the LGA moves with respect to the spacecraft's center of mass, therefore generating a large Doppler signal which must be removed (Barbaglio et al., 2012). As a result, the location of the phase center of this antenna with respect to Cassini's center of mass must be included in the estimation process.

An important issue in the analysis of Cassini's gravity data is the need to update Titan's ephemeris. During a typical Titan flyby, Cassini's positioning with respect to Titan has a formal uncertainty of at most a few meters in the radial direction during Titan's closest approach (according to orbital geometry and available data). The analysis of the first four passes (Iess et al., 2010) was carried out by adopting the latest ephemeris available from the navigation team whereas the first six gravity passes (Iess et al., 2012) were analyzed by updating Saturnian ephemerides (in particular Titan) in order to match the observed Doppler data with Titan's updated ephemeris and gravity field. The updated ephemerides had to be dynamically consistent between all the passes. The inclusion of the latest data does not allow us to obtain a satisfactory fit of the Doppler data together with an update of Titan's ephemeris. This may be related to the need for a more complete dynamical model for the Saturnian satellites due to the long timespan covered by Cassini's observations (10 years). The inclusion of effects such as Saturn's tidal dissipation (Fuller et al., 2016) or frequency dependent tides, Saturn's normal modes, or an accurate modelling of Saturn's pole motion may be crucial to obtain a dynamically consistent motion of Saturn's satellites to the level of accuracy required by the data. Thus, the approach taken hereafter is to update Titan's ephemeris for each of Cassini's passes, without requiring the updated ephemeris to be dynamically coherent between the passes. This approach allows us to overcome the difficulties inherent to requiring a global ephemeris, allowing for deviations in Titan's orbit. Of course, the larger number of estimated parameters implies a penalty on the uncertainties of the gravity coefficients. We use the latest available Saturn's satellite ephemerides, SAT389 (see NASA's Navigation and Ancillary Information Facility website), as a reference ephemeris. Titan's position has an a priori uncertainty of $5 \mathrm{~m}$ in the radial direction, and 50 and $500 \mathrm{~m}$ in the along-track and out-of-plane directions (Boone et al., 2017). The limited dataset cannot provide a more accurate estimate of Titan's orbit. The required corrections are of the same order of magnitude as the assumed a priori uncertainties. Our reconstruction of Titan's orbit is valid only in the timeframe of each arc with the intent of obtaining an

\footnotetext{
${ }^{1}$ Consider parameters are those which are not estimated but whose a priori uncertainty contributes to the covariance matrix.
} 
Table 1

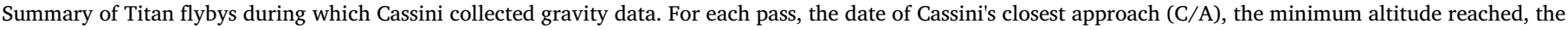

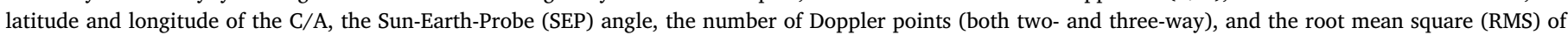
Doppler residuals at $60 \mathrm{~s}$ are reported.

\begin{tabular}{|c|c|c|c|c|c|c|c|}
\hline & Date of C/A & Altitude & Latitude & Longitude & SEP angle & Number of Doppler points & RMS of Doppler residuals (at $60 \mathrm{~s}$ ) \\
\hline T011 & 27 Feb. 2006 & $1812 \mathrm{~km}$ & $0.2^{\circ} \mathrm{S}$ & $255.6^{\circ} \mathrm{E}$ & $150.2^{\circ}$ & 1051 & $0.017 \mathrm{~mm} / \mathrm{s}$ \\
\hline T022 & 28 Dec. 2006 & $1297 \mathrm{~km}$ & $45.4^{\circ} \mathrm{N}$ & $355.9^{\circ} \mathrm{E}$ & $130.6^{\circ}$ & 1151 & $0.024 \mathrm{~mm} / \mathrm{s}$ \\
\hline T033 & 29 June 2007 & $1933 \mathrm{~km}$ & $8.4^{\circ} \mathrm{N}$ & $63.1^{\circ} \mathrm{E}$ & $46.4^{\circ}$ & 1508 & $0.028 \mathrm{~mm} / \mathrm{s}$ \\
\hline T045 & 31 July 2008 & $1614 \mathrm{~km}$ & $43.5^{\circ} \mathrm{S}$ & $162.7^{\circ} \mathrm{E}$ & $30.0^{\circ}$ & 1288 & $0.068 \mathrm{~mm} / \mathrm{s}$ \\
\hline T068 & 20 May 2010 & $1397 \mathrm{~km}$ & $48.9^{\circ} \mathrm{S}$ & $241.1^{\circ} \mathrm{E}$ & $120.3^{\circ}$ & 2226 & $0.025 \mathrm{~mm} / \mathrm{s}$ \\
\hline T074 & 18 Feb. 2011 & $3651 \mathrm{~km}$ & $1.0^{\circ} \mathrm{N}$ & $113.4^{\circ} \mathrm{E}$ & $131.2^{\circ}$ & 1847 & $0.063 \mathrm{~mm} / \mathrm{s}$ \\
\hline T089 & 17 Feb. 2013 & $1978 \mathrm{~km}$ & $21.0^{\circ} \mathrm{N}$ & $203.1^{\circ} \mathrm{E}$ & $106.0^{\circ}$ & 1479 & $0.033 \mathrm{~mm} / \mathrm{s}$ \\
\hline T099 & 06 Mar. 2014 & $1500 \mathrm{~km}$ & $31.1^{\circ} \mathrm{S}$ & $181.0^{\circ} \mathrm{E}$ & $111.0^{\circ}$ & 1860 & $0.022 \mathrm{~mm} / \mathrm{s}$ \\
\hline $\mathrm{T} 110$ & 16 Mar. 2015 & $2274 \mathrm{~km}$ & $74.8^{\circ} \mathrm{N}$ & $263.1^{\circ} \mathrm{E}$ & $108.6^{\circ}$ & 1836 & $0.064 \mathrm{~mm} / \mathrm{s}$ \\
\hline T122 & 10 Aug. 2016 & $1698 \mathrm{~km}$ & $12.4^{\circ} \mathrm{N}$ & $234.4^{\circ} \mathrm{E}$ & $112.7^{\circ}$ & 1858 & $0.061 \mathrm{~mm} / \mathrm{s}$ \\
\hline
\end{tabular}

a

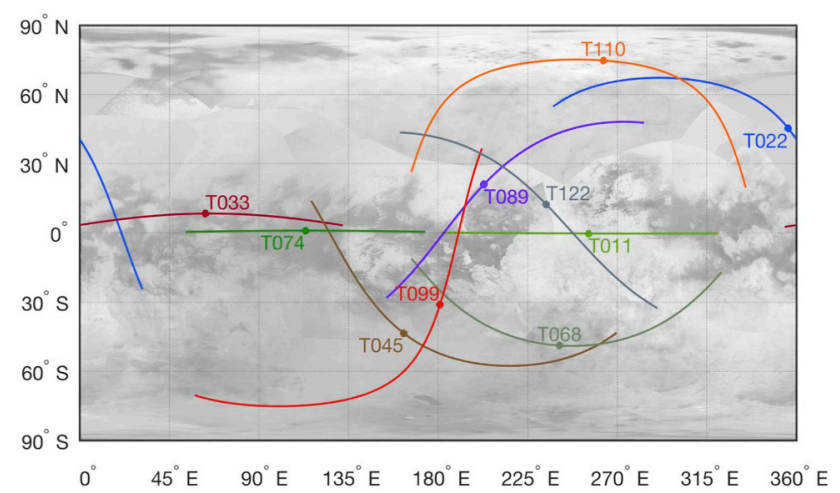

b

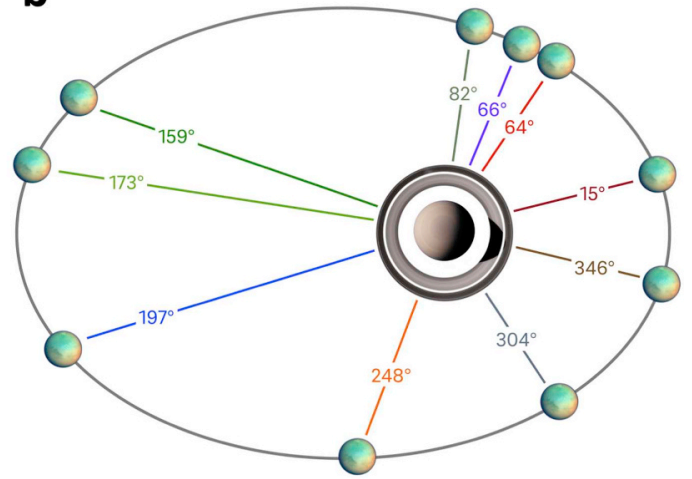

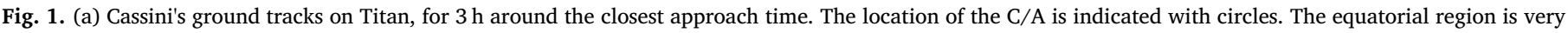

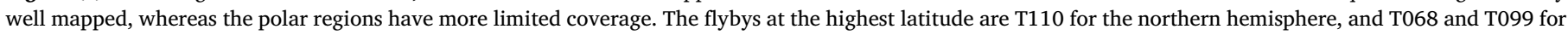

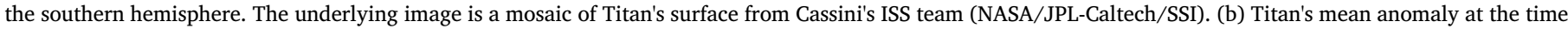
of Cassini's closest approach. A good sampling is crucial for the determination of Titan's tidal response.

unbiased estimate of the gravity field.

\section{Gravity field results}

Titan's gravitational potential, evaluated at radius $r$, colatitude $\theta$, and longitude $\phi$, can be expressed as a linear combination of spherical harmonic functions as

$U(r, \theta, \phi)=-\frac{G M}{r} \sum_{l=0}^{\infty} \sum_{m=0}^{l}\left(\frac{R_{\mathrm{ref}}}{r}\right)^{l}\left(C_{l m} \cos m \phi+S_{l m} \sin m \phi\right) P_{l m}(\cos \theta)$

where $C_{l m}$ and $S_{l m}$ represent the unnormalized degree- $l$ and order- $m$ dimensionless gravitational potential coefficients at some reference radius (which we take to be $R_{\text {ref }}=2575 \mathrm{~km}$ ), and where $P_{l m}(\cos \theta)$ are the unnormalized associated Legendre functions. $G$ is the universal gravitational constant and $M$ is Titan's mass. Note that all $S_{l 0}=0$ and that $C_{00} \equiv 1$. Because the gravitational potential is resolved about the center of mass, the degree- 1 terms are all zero.

The gravity field coefficients estimated for Titan are reported in Table 2 along with Titan's tidal Love number $k_{2}$ whereas Fig. 2 reports the root mean square of the fully normalized gravity field coefficients $C_{l}$, showing that the power spectrum of the gravity coefficients is larger than the uncertainty up to degree 5. Indeed, truncating the gravity field expansion to a smaller degree cannot provide a good fit of all Doppler data. On the other hand, higher degrees cannot be determined with sufficient accuracy and have been neglected. However, only a few coefficients are determined with good accuracy, such as the degree 2 hydrostatic terms and the sectorial harmonics of degree 3 and 4. Still, an expansion up to degree and order 5 of the gravity field is an improvement with respect to the previous solution (Iess et al., 2012),
Table 2

Estimated values and 1- $\sigma$ uncertainties for Titan's gravitational parameter (GM), unnormalized dimensionless gravitational potential coefficients (where $J_{l}=-C_{l 0}$ ) corresponding to a reference radius of $2575 \mathrm{~km}$, and the tidal Love number $k_{2}$ for the reference solution.

\begin{tabular}{crrr}
\hline$G M\left(\mathrm{~km}^{3} / \mathrm{s}^{2}\right)$ & \multicolumn{1}{c}{$8978.1383 \pm 0.0003$} & & \\
\hline$J_{2}\left(\times 10^{6}\right)$ & $33.089 \pm 0.609$ & & \\
$C_{21}\left(\times 10^{6}\right)$ & $0.513 \pm 0.215$ & $S_{21}\left(\times 10^{6}\right)$ & $0.612 \pm 0.359$ \\
$C_{22}\left(\times 10^{6}\right)$ & $10.385 \pm 0.084$ & $S_{22}\left(\times 10^{6}\right)$ & $-0.064 \pm 0.066$ \\
$J_{3}\left(\times 10^{6}\right)$ & $-0.179 \pm 0.720$ & & \\
$C_{31}\left(\times 10^{6}\right)$ & $1.481 \pm 0.254$ & $S_{31}\left(\times 10^{6}\right)$ & $0.811 \pm 0.402$ \\
$C_{32}\left(\times 10^{6}\right)$ & $0.183 \pm 0.153$ & $S_{32}\left(\times 10^{6}\right)$ & $-0.027 \pm 0.099$ \\
$C_{33}\left(\times 10^{6}\right)$ & $-0.222 \pm 0.017$ & $S_{33}\left(\times 10^{6}\right)$ & $-0.226 \pm 0.019$ \\
$J_{4}\left(\times 10^{6}\right)$ & $-1.077 \pm 1.844$ & & \\
$C_{41}\left(\times 10^{6}\right)$ & $-0.842 \pm 0.299$ & $S_{41}\left(\times 10^{6}\right)$ & $0.191 \pm 0.717$ \\
$C_{42}\left(\times 10^{6}\right)$ & $0.183 \pm 0.107$ & $S_{42}\left(\times 10^{6}\right)$ & $0.198 \pm 0.106$ \\
$C_{43}\left(\times 10^{6}\right)$ & $-0.012 \pm 0.039$ & $S_{43}\left(\times 10^{6}\right)$ & $-0.062 \pm 0.033$ \\
$C_{44}\left(\times 10^{6}\right)$ & $-0.014 \pm 0.003$ & $S_{44}\left(\times 10^{6}\right)$ & $-0.012 \pm 0.004$ \\
$J_{5}\left(\times 10^{6}\right)$ & $1.118 \pm 2.022$ & & \\
$C_{51}\left(\times 10^{6}\right)$ & $0.361 \pm 0.406$ & $S_{51}\left(\times 10^{6}\right)$ & $0.267 \pm 0.604$ \\
$C_{52}\left(\times 10^{6}\right)$ & $-0.097 \pm 0.118$ & $S_{52}\left(\times 10^{6}\right)$ & $0.044 \pm 0.094$ \\
$C_{53}\left(\times 10^{6}\right)$ & $-0.016 \pm 0.019$ & $S_{53}\left(\times 10^{6}\right)$ & $-0.004 \pm 0.012$ \\
$C_{54}\left(\times 10^{6}\right)$ & $0.007 \pm 0.004$ & $S_{54}\left(\times 10^{6}\right)$ & $-0.002 \pm 0.004$ \\
$C_{55}\left(\times 10^{6}\right)$ & $0.000 \pm 0.001$ & $S_{55}\left(\times 10^{6}\right)$ & $0.000 \pm 0.001$ \\
\hline$k_{2}$ & $0.616 \pm 0.067$ & &
\end{tabular}

which resolved Titan's gravity field only up to degree 3 (SOL1a and SOL2) or degree 4 (SOL1b). The uncertainties on the gravity field coefficients reported in Table 2 are generally up to a factor of 2 larger than those reported by less et al. (2012), even if a broader dataset has been used. This apparent discrepancy lies in the complexity of the 


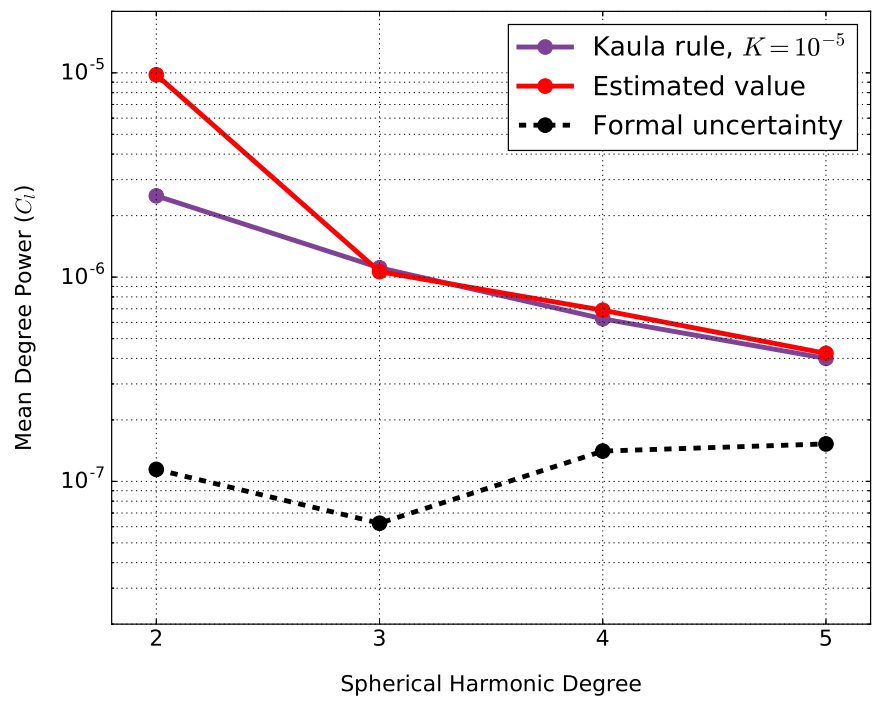

Fig. 2. Root mean square of the fully normalized gravity coefficients, $C_{l}=\sqrt{\frac{\sum_{m}\left(\bar{C}_{l m}^{2}+\bar{S}_{l m}^{2}\right)}{2 l+1}}$. The values and 1- $\sigma$ uncertainties are reported and compared to a Kaula's rule, $C_{l}=K / l^{2}$, with $K=10^{-5}$.

adopted dynamical model: firstly, the maximum rank of our gravity solution is larger, meaning that more coefficients are included in the estimation process; secondly, the approach selected for Titan's ephemeris is different, with our approach leading to about a $20 \%$ increase of the uncertainties, especially in the low-degree field. The tidal Love number $k_{2}$, however, is determined with a better accuracy due to the broader sampling in longitude of Titan's mean anomaly.

Fig. 2 plots the power law $C_{l}=K / l^{2}$, known as the Kaula rule (Kaula, 1963), with $K=10^{-5}$, showing good agreement with the estimated power of Titan's gravity field for degrees larger than $2\left(J_{2}\right.$ and $C_{22}$ are dominated by the hydrostatic component). The Kaula rule is an empirical law able to represent the power spectrum of the gravitational field of rocky planets, but it has never been observed for an icy satellite like Titan. Note that a Kaula constraint has not been imposed into the estimation process. This empirical rule conveys very little information about the physical nature of the density anomalies responsible for the field, since a power law like this can arise in a number of physically plausible ways and is seen for all terrestrial bodies (including Earth) despite the substantial differences among these bodies that are known to exist by other methods, most obviously the fact that Earth has plate tectonics. A fit of the gravity coefficients (excluding degree 2 ) yields $C_{l}=0.97 \times 10^{-5} / l^{2}$. The coefficient $\left(K \approx 10^{-5}\right)$ might convey more physical information than the spectral slope since it is affected by the degree of compensation, the volume of the region responsible for the density anomalies, and the typical stress levels that exist within the body. For example, if we suppose that there is a peak deviatoric stress that scales as $\Delta \rho \times g \times R$ (where $\Delta \rho$ is a typical density anomaly, $g$ is gravitational acceleration, and $R$ is radius or some comparable large length scale) then we might expect $C_{l} \propto(R \times g)^{-1} \propto g^{-2}$ since $g$ scales as $R$. Note however that this scaling should not be used when going from rocky bodies to icy bodies since the expected peak stresses are lower in ice and the mean density is lower, so the partial success of this scaling for terrestrial bodies does not imply it should work for icy bodies. Recently, Ermakov et al. (2018) reviewed the Kaula rule of different terrestrial bodies in the literature to achieve a more general scaling rule among them. Their result for the gravity spectra is $C_{l} \propto g^{-1.72}$. However, if we were to apply this suggested rescaling rule to Titan, we obtain an overestimate of Titan's gravity spectrum by a factor of 30 to 50 (Titan's surface gravity is 0.14 times Earth's gravity). This is not surprising considering the large degree of compensation (discussed further below), the closely related likelihood of a rather thin outer shell of ice (which reduces the volume in which the relevant density anomalies arise), and the lower strength of ice (which reduces the peak deviatoric stresses that are assumed to be universal in the scaling argument given above). Unfortunately, Titan is the only icy satellite whose gravity field has been confidently resolved up to degree 5 , and a comparison within this class of bodies is not possible at present. Note also that terrestrial planets as a group are actually much more similar to each other (in respect of a core and mantle structure) than the large icy satellites, and that Europa, a body of much current interest, is in neither class (it has far less ice than rock).

The direction of Titan's principal axes of inertia can be inferred from the degree 2 coefficients. For a synchronous rotator, the axis of least inertia is expected to be aligned approximately with the empty focal point of Titan's orbit around Saturn (Murray and Dermott, 1999). A small 3-2-1 rotation can be defined to relate the body-fixed reference frame (a frame whose $\mathrm{z}$-axis aligns with the spin pole and $\mathrm{x}$-axis aligns with the prime meridian; we adopted the frame defined in Meriggiola et al., 2016) to the principal axes reference frame. The three rotations are related to the fully normalized gravity coefficients (e.g., Liu and Chao, 1991) by:

$$
\begin{aligned}
& \epsilon_{x}=-\frac{\bar{S}_{21}}{\sqrt{3} \bar{C}_{20}+\bar{C}_{22}}=2.85^{\circ} \pm 1.67^{\circ} \\
& \epsilon_{y}=\frac{\bar{C}_{21}}{\sqrt{3} \bar{C}_{20}-\bar{C}_{22}}=-0.55^{\circ} \pm 0.23^{\circ} \\
& \epsilon_{z}=\frac{\bar{S}_{22}}{2 \bar{C}_{22}}=-0.18^{\circ} \pm 0.18^{\circ}
\end{aligned}
$$

all compatible with zero at most at 3- $\sigma$. The direction of the axis of least inertia is compatible at $1-\sigma$ with the expectation for a synchronous rotator.

Due to the non-negligible eccentricity of Titan's orbit $(e=0.028)$, tides raised by Saturn change with time, producing a variable quadrupole component. Titan's tidal response to Saturn can be described by its $k_{2}$ tidal Love number, which is crucial to understanding the interior structure of Titan. Cassini's flybys dedicated to gravity science occurred at different Titan mean anomalies (see Fig. 1b), probing the variable quadrupole field at different locations, and providing a very accurate measurement of its degree- 2 tidal Love number. The best estimate of Titan's tidal response is $k_{2}=0.616 \pm 0.067$, which is within $1-\sigma$ of the all the three solutions given by Iess et al. (2012), but has a smaller uncertainty due to the larger data set used for its estimation. However, the accuracy on $k_{2}$ is somehow degraded due to the local ephemeris approach used for Titan's orbit (a covariance analysis shows a degradation of about $20 \%$ in the uncertainty with respect to a global Titan ephemeris). However, the consistency with the previous solution confirms Titan's large deformability over timescales of its orbital period, consistent with a global ocean beneath an outer ice shell that is sufficiently thin that it does not greatly impede the deformation that would be expected for a hydrostatic fluid overlying the core. The implications of this value are discussed further in Section 4.

The reference solution does not account for the tidal dissipation, and only the real component of the Love number $k_{2}$ is estimated. By including the time lag into the tidal model, the gravity coefficients remain compatible at 1- $\sigma$ with our reference solution. The estimate for the time lag is $\Delta t=14,000 \pm 20,000 \mathrm{~s}$, which corresponds to an angular lag angle of $\delta=3.7^{\circ} \pm 5.4^{\circ}$ or to an imaginary part of the complex Love number of $k_{2}{ }^{i m}=0.082 \pm 0.118$. The estimated quality factor can be inferred from the real and imaginary part of the tidal Love number $k_{2}$ from $Q=k_{2}{ }^{\mathrm{re}} / k_{2}{ }^{\mathrm{im}}$. The inverse of the quality factor is $1 /$ $Q=0.133 \pm 0.192$, implying $Q \approx 7.6$. The time lag is compatible with zero at $1-\sigma$, thus we can draw no strong conclusions about the internal dissipation from Cassini gravity data.

The robustness of our reference solution has been tested, with the aim of assessing whether the formal uncertainties we quoted in Table 2 are statistically meaningful and to confirm that biases in the estimated 

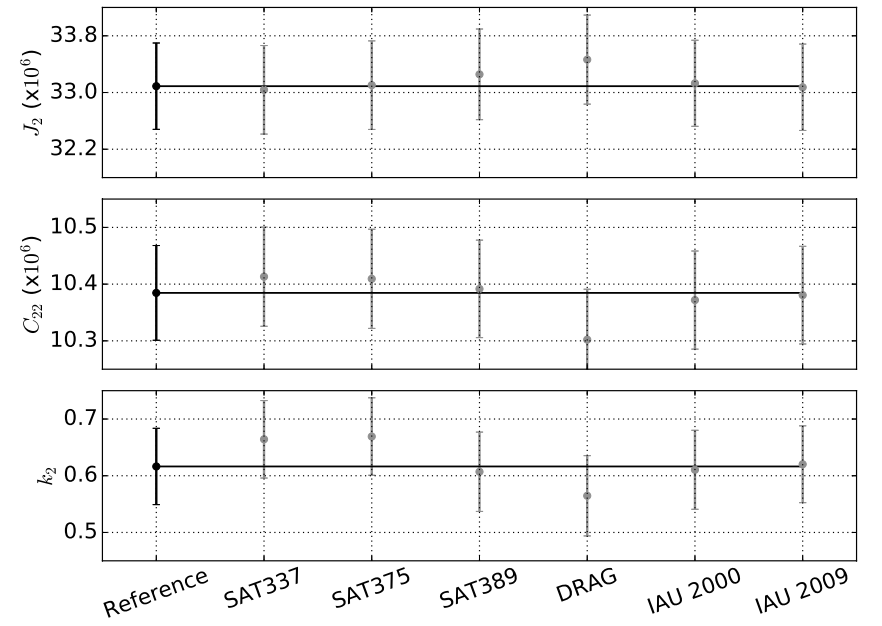

Fig. 3. Comparison of estimated values and 1- $\sigma$ uncertainties for $J_{2}, C_{22}$, and $k_{2}$ between our reference solution and other solutions: SAT337 and SAT375 update a different version of Saturn's satellite ephemerides, whereas SAT389 has 3-times larger a priori uncertainty for Titan's position and velocity; DRAG estimate a drag coefficient for each of the low-altitude passes instead of Titan's base density and scale height; IAU 2000 and IAU 2009 adopt the rotation models suggested by IAU.

values are absent. Since the selection of the reference ephemeris and the a priori covariance given to Titan are crucial in our approach, a robustness test has been performed by starting from older versions of the ephemerides (e.g., SAT337 and SAT375) or by enlarging the a priori covariance by a factor of 3 (see Fig. 3). All the solutions are statistically consistent at 1- $\sigma$, confirming the robustness of Titan's gravity field solution with respect to a change in Titan's ephemeris. Concerning Titan's atmosphere, we verified that the estimation of drag coefficients in lowaltitude flybys instead of Titan's base density and scale height yields a statistically compatible solution (within 1- $\sigma$, see Fig. 3 ). We tested also the impact of the assumed rotational model by adopting rotation models advised by the International Astronomical Union. The models given in Seidelmann et al. (2002) and that proposed by Stiles, et al. (2008) and later adopted by IAU (Archinal et al., 2011; Archinal et al., 2018) give gravity fields statistically consistent at 1- $\sigma$ with our reference solution (see Fig. 3).

\section{Implications for Titan's interior}

Titan's gravitational field conforms closely to the expectation for a body that has relaxed to hydrostatic equilibrium, with a moment of inertia factor in the vicinity of 0.341 (Fig. $4 \mathrm{a}$; see below). The moment of inertia factor is given by $I / M R^{2}$, where $I$ is the mean moment of inertia, $M$ is Titan's mass, and $R$ is Titan's mean radius. For a relaxed hydrostatic body, the departure from spherical symmetry arises mainly from rotational and tidal deformation and the associated redistribution of mass gives rise to the large $J_{2}$ and $C_{22}$ terms in the gravitational potential. For a relaxed hydrostatic body, these terms have a theoretical ratio of $J_{2} / C_{22}=10 / 3$, to first order (the dashed line in Fig. 4a). The moment of inertia values indicated along the dashed line in Fig. 4a can be computed via the Radau-Darwin equation (Darwin, 1899; Murray and Dermott, 1999), which relates the moment of inertia factor to the fluid Love number $k_{2 f}$, which can in turn be related to $J_{2}$ and $C_{22}$ (e.g., see eqs. 16, 21-23 in Hemingway et al., 2018). If Titan were perfectly hydrostatic, one could thus obtain $k_{2 f}$, and therefore the moment of inertia factor, directly from either of the $J_{2}{ }^{\text {obs }}$ or $C_{22}{ }^{\text {obs }}$ terms, where the superscript 'obs' refers to the observed values (Table 2). Because of the departure from the hydrostatic expectation, however, $J_{2}{ }^{\text {obs }}$ and $C_{22}{ }^{\text {obs }}$ correspond to slightly inconsistent moment of inertia factors. In order to approximately satisfy both observations, with no preference for one over the other, we therefore find the moment of inertia factor that yields, via the Radau-Darwin relation (see references above), hydrostatic values $J_{2}{ }^{\text {hyd }}$ and $C_{22}$ hyd that minimize $\sqrt{\left(J_{2}^{\text {obs }}-J_{2}^{\text {hyd }}\right)^{2}+\left(C_{22}^{\text {obs }}-C_{22}^{\text {hyd }}\right)^{2}}$. The resulting best fitting moment of inertia factor is approximately 0.341 , corresponding to a potential fluid Love number, $k_{2 f}$, of approximately 1.01 , with $J_{2}^{\text {hyd }}=33.21 \times 10^{-6}$ and $C_{22}^{\text {hyd }}=9.96 \times 10^{-6}$ (cf. Table 2).

Note that, whereas for fast spinning bodies like Enceladus, the hydrostatic ratio becomes slightly smaller than $10 / 3$ as higher order corrections become important (Tricarico, 2014), the first order approximation of $10 / 3$ is highly accurate for Titan (to within $0.02 \%$ ). Another correction can account for Titan's orbital eccentricity (Matsuyama, 2011), giving a correction smaller than $0.3 \%$. Thus, we can state that for a hydrostatic Titan, the value of $10 / 3$ is correct to within $0.3 \%$, ten times below the current accuracy. Our estimate of the ratio is $J_{2} / C_{22}=3.186 \pm 0.077$, compatible with the hydrostatic value at $2-\sigma$. Though, as we discuss further below, having the $10 / 3$ ratio is a necessary, but not sufficient condition for hydrostatic equilibrium.

In the simplest terms, Titan may be regarded as a mainly rocky core surrounded by an $\mathrm{H}_{2} \mathrm{O}$ mantle consisting of various solid and liquid phases. Adopting a simple two-layer model (rocky core, $\mathrm{H}_{2} \mathrm{O}$ mantle), taking the moment of inertia factor to be 0.341 , and assuming the average density across the $\mathrm{H}_{2} \mathrm{O}$ layers is $1000 \mathrm{~kg} / \mathrm{m}^{3}$, the known radius $(2575 \mathrm{~km})$ and bulk density $\left(1881 \mathrm{~kg} / \mathrm{m}^{3}\right)$ can be used to solve for the core radius and density (e.g., see eqs. 1 and 2 in Hemingway et al., 2018). Under these assumptions, we obtain an $\mathrm{H}_{2} \mathrm{O}$ layer thickness of $\sim 400 \mathrm{~km}$, leaving a large rocky core with a density around $2500 \mathrm{~kg} / \mathrm{m}^{3}$. For the sake of generality, we carry out this calculation over a wide range of different moments of inertia and $\mathrm{H}_{2} \mathrm{O}$ layer densities (Fig. 5), allowing for the possibility of, for example, the higher ocean densities permitted by the large tidal $k_{2}$ (Iess et al., 2012; Stiles, et al., 2008; Meriggiola et al., 2016; and see below). Such higher density outer layers would imply a significantly smaller and denser core, even when adopting the same moment of inertia. Moreover, the possibility of a non-hydrostatic interior (see below) permits moments of inertia smaller than 0.341 , corresponding to still smaller and denser cores (Fig. 5).

The measured $J_{2}$ and $C_{22}$ tell us that the hydrostatic, or fluid, $k_{2}$ Love number is close to unity (see above). This is substantially larger than the tidal $k_{2}$, which tells us that some major portion of Titan does not behave like a fluid at tidal timescales. The value for the tidal $k_{2}$ for a thin shell and rigid interior beneath the ocean consistent with the moment of inertia is about 0.42 (Rappaport et al., 2008; Iess et al., 2012). This assumes that the outermost shell has little effect on the response; any effect it could have because of its elasticity would only decrease the predicted $k_{2}$. It follows that there are only two candidates for increasing the theoretical value to the observed value: either increase the ocean density (which also decreases the core size to be compatible with the moment of inertia) or allow the core to have a significant non-elastic (i.e., viscous) response at tidal timescales. Some combination of these two is possible of course. The interpretation is non-unique but the choice of a viscous core response (which is discussed in Iess et al., 2012) requires a non-Maxwell like response since a Maxwell model that simultaneously increases the real part of $k_{2}$ also increases the imaginary part to a level inconsistent with the data. The core need not exhibit a Maxwell rheology, so this possibility is not excluded. As discussed by Iess et al. (2012) and considered by Rappaport et al. (2008) and Baland et al. (2014), an ocean density of $1200-1300 \mathrm{~kg} / \mathrm{m}^{3}$ is cosmochemically possible and yields a $k_{2}$ of 0.55 to 0.6 . We lack sufficient understanding of the rheology of deeper regions to assess quantitatively the possible contribution of a non-elastic response for that region. It is also possible that the outer shell is reducing the Love number (which makes it harder to explain the result we obtained with a dense ocean alone) and a dynamic response to the tidal perturbation has also been suggested. Most interest in dynamic tides has centered on the possibility of additional tidal heating (Matsuyama 

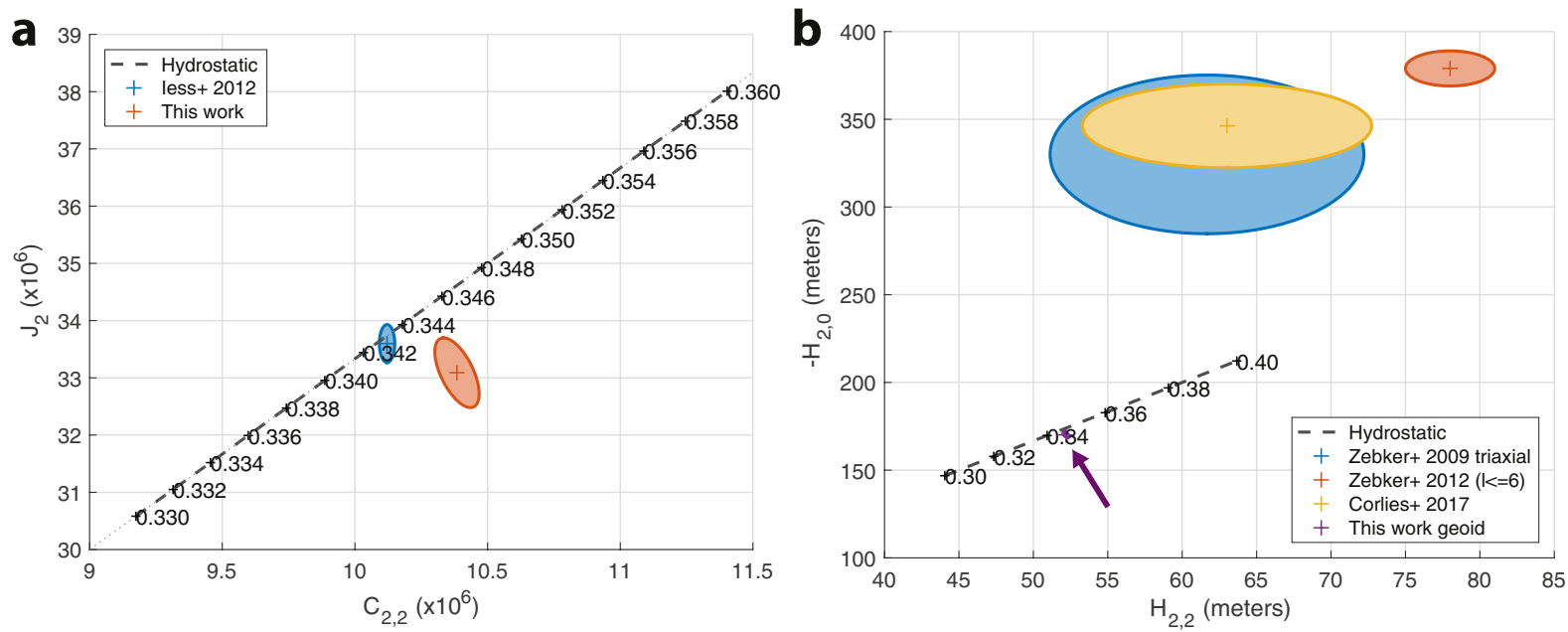

Fig. 4. (a) The newly measured (red) dominant quadrupole gravitational potential coefficients, $J_{2}$ and $C_{22}$, in comparison to the results of Iess et al. (2012) (blue), with 1- $\sigma$ uncertainty ellipses, and (b) the corresponding degree- 2 terms of Titan's figure, $-H_{20}$ and $H_{22}$, in relation to the measured geoid (small purple ellipse, highlighted by purple arrow); three distinct shape models are shown for comparison (blue: the triaxial ellipsoidal solution of Zebker et al., 2009; red: the $l \leq 6$ solution of Zebker et al., 2012; and yellow: Corlies et al., 2017). In both panels, the observations are shown in relation to the expectation for hydrostatic equilibrium (dashed line, with numbers indicating normalized mean moments of inertia, computed via the Radau-Darwin relation). Whereas the shape departs substantially from the expectation for hydrostatic equilibrium, the gravitational field departs only slightly from the hydrostatic expectation. (For interpretation of the references to color in this figure legend, the reader is referred to the web version of this article.)

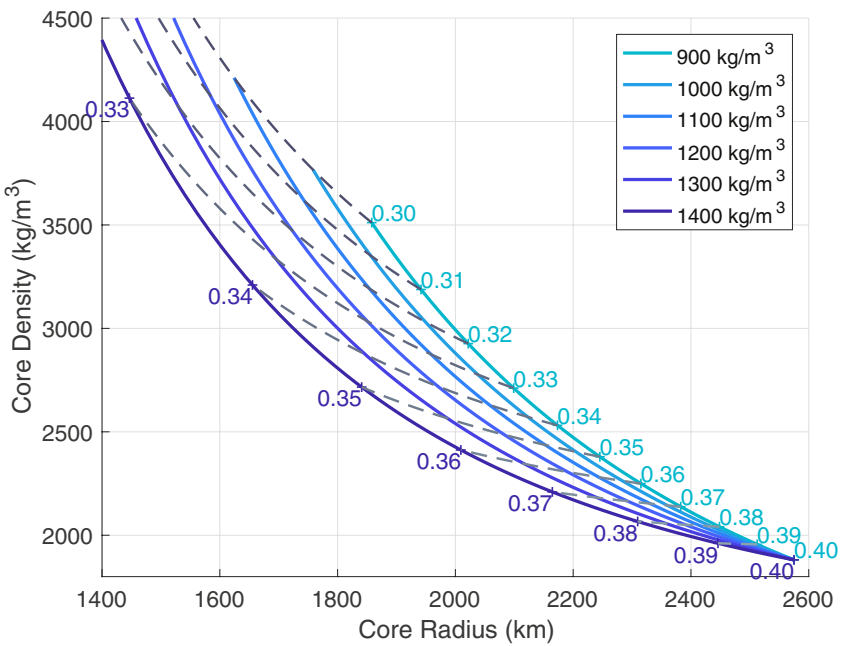

Fig. 5. Range of possible core radii and densities in relation to a range of moments of inertia (constant along dashed curves) assuming various mean densities for the $\mathrm{H}_{2} \mathrm{O}$ mantle (solid curves). A two-layer body is assumed (rocky core, $\mathrm{H}_{2} \mathrm{O}$ mantle) and is subject to the constraints of Titan's known total radius $(2575 \mathrm{~km})$ and bulk density $\left(1881 \mathrm{~kg} / \mathrm{m}^{3}\right)$.

et al., 2018), which is not desirable for explaining Titan, but non-resonant dynamic tides might be responsible for a significant contribution to the Love number.

Unlike its gravitational field, Titan's figure (Zebker et al., 2009; Corlies et al., 2017) departs substantially from the hydrostatic expectation, exhibiting considerable excess flattening. Using the latest shape model (Corlies et al., 2017), the dominant degree-2 terms have the ratio $-H_{20} / H_{22}=5.5 \pm 1.2$, far larger than the hydrostatic value of 3.333 (Fig. 4b). As with the gravitational potential, we compute the hydrostatic equilibrium figures, shown along the dashed line in Fig. 4b for various moments of inertia, again using the Radau-Darwin relation (e.g., see eqs. 18, 21-23 in Hemingway et al., 2018). Whereas Fig. 6b shows the Corlies et al. (2017) topography with respect to a reference sphere, with radius $2575 \mathrm{~km}$, Fig. 6d shows the topography with respect to the hydrostatic equilibrium figure expected for an assumed moment of inertia factor of 0.341 . Because the measured gravitational potential is so close to the hydrostatic expectation, this hypothetical hydrostatic equilibrium figure is nearly identical to the geoid (small purple ellipse in Fig. 4b). The geoid is a surface where the potential, including tidal and rotational effects, has a constant value of $G M / R_{\text {ref. }}$ The geoid can be approximated to first order as

$$
\begin{aligned}
N(\theta, \phi)= & R_{\text {ref }} \sum_{l=0}^{\infty} \sum_{m=0}^{l}\left(C_{l m} \cos m \phi+S_{l m} \sin m \phi\right) P_{l m}(\cos \theta) \\
& -\frac{5 \omega^{2} R_{r e f}^{2}}{6 g} P_{20}(\cos \theta)+\frac{\omega^{2} R_{r e f}^{2}}{4 g} P_{22}(\cos \theta) \cos 2 \phi
\end{aligned}
$$

where the last two terms are required to account for the combined tidal and rotational potentials and where $\omega$ and $g$ are Titan's rotation rate and surface gravity, respectively. We do not show the topography with respect to the geoid because the result is visually indistinguishable from the non-hydrostatic topography shown in Fig. 6d. Thus, measured with respect to the geoid, or almost equivalently, to a hydrostatic equilibrium figure with moment of inertia factor 0.341 , Titan's topography is relatively high in low latitudes-a notable exception being the topographic low near the Xanadu province-and is lowest near the poles, especially on the trailing hemisphere (Fig. 6d).

The non-hydrostatic topography complicates the simple picture above because it should also contribute to the gravitational field, and yet there appears to be no significant non-hydrostatic gravity, at least at degree 2 (Fig. 4a). This is a strong indication that the $>500 \mathrm{~m}$ of nonhydrostatic topography are highly compensated, meaning that the gravity anomalies associated with this topography must be offset by internal density anomalies. This is expected in the limit of a weak lithosphere, because the topography can only be supported isostatically (i.e., by buoyancy). The presence of a liquid water ocean beneath the floating ice shell provides a natural mechanism for Airy-type isostatic compensation in the sense of lateral shell thickness variations (e.g., Nimmo and Bills, 2010) wherein the low density isostatic roots offset the effect of the mass corresponding to the surface topography. It has also been suggested that Pratt-type isostatic compensation could play a role in the form of hydrocarbon-cycle-related density anomalies near Titan's polar regions (Choukron and Sotin, 2012), which would likewise reduce the contribution to the measured gravity field made by the nonhydrostatic topography. 

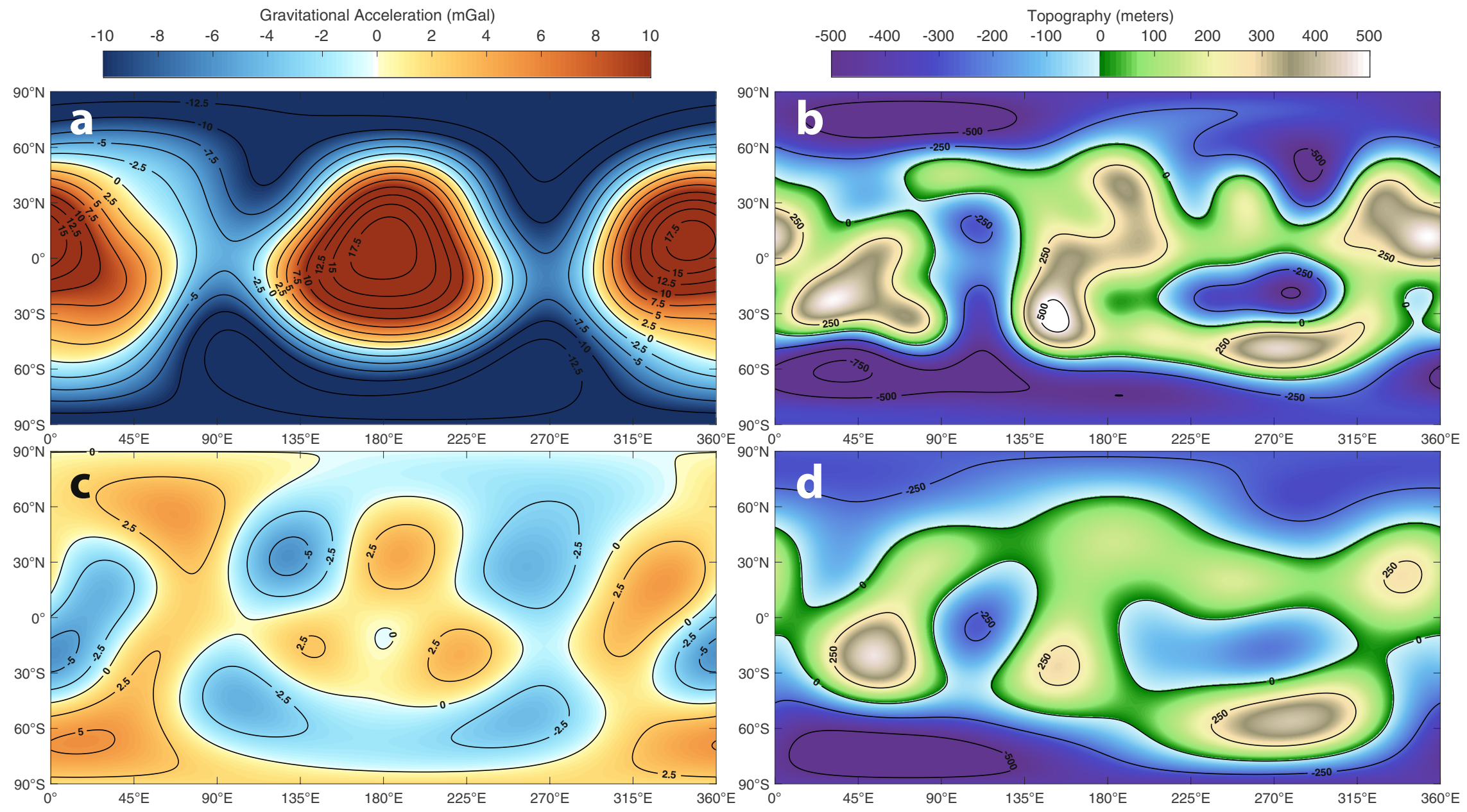

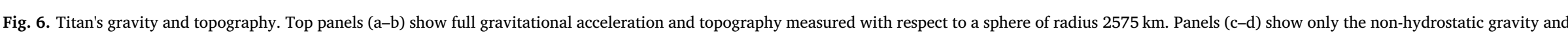
topography (i.e., departures from the hydrostatic expectation, where the hydrostatic component is computed via the Radau-Darwin relation) where we have assumed a moment of inertia factor of 0.341. 

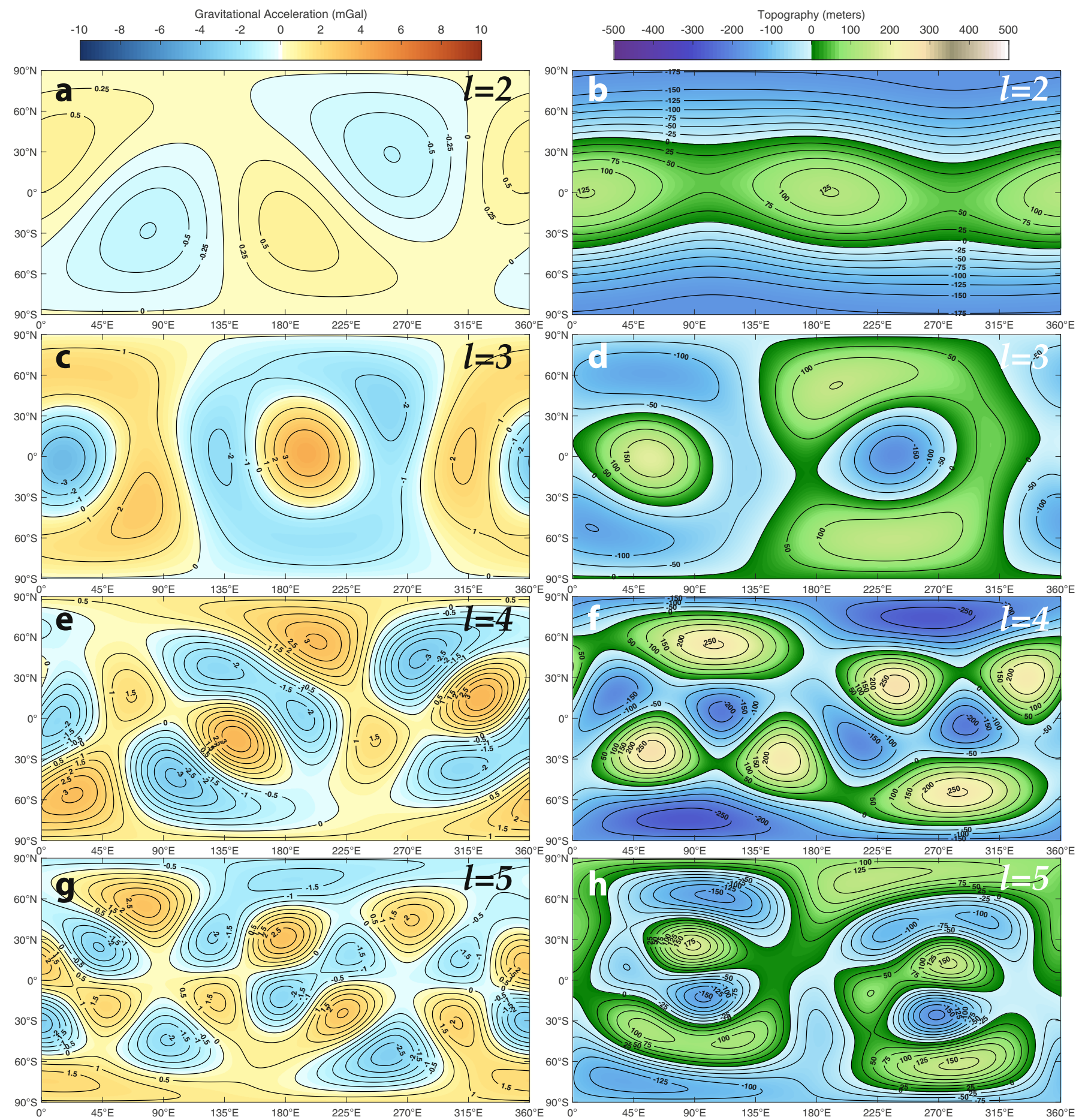

Fig. 7. Non-hydrostatic gravity (left column) and topography (right column) for degrees 2-5. Whereas the signals beyond degree 2 (c-h) are independent of the assumed moment of inertia, the degree-2 signal (a-b) required subtracting the contributions from a hydrostatic body with moment of inertia factor 0.341 .

Even in the limit of perfect isostatic equilibrium, however, the topography's contribution to the gravity signal should generally not be zero because of the finite thickness of the ice shell, which attenuates the compensating effect. Indeed, the relationship between the non-hydrostatic gravity and topography, typically quantified by the spectral admittance and correlation (e.g., Wieczorek, 2015; our Fig. 8), can often be used to estimate the compensation depth (i.e., the ice shell thickness). In the present case, however, Titan's non-hydrostatic topography does not contribute strongly to the non-hydrostatic gravity-the admittance and correlation spectra are compatible with zero (purple line in Fig. 8). That is, while some non-hydrostatic gravity is evident, at least beyond degree 2 (Fig. 7, left column), it is not spatially well correlated with the non-hydrostatic topography (Fig. 7, right column), precluding direct quantification of the compensation depth via a spectral admittance analysis and therefore a meaningful estimate of the ice shell thickness, at least on the basis of gravity and topography data alone.

This lack of spatial correlation could be an indication that the ice shell is very thin, making isostatic compensation very effective in reducing the non-hydrostatic gravity. Alternatively, the topography could be over-compensated. That is, the isostatic roots may be larger than what would be expected in the limit of isostatic equilibrium. This could be the case if the ice shell thickness variations arise from thinning/ 

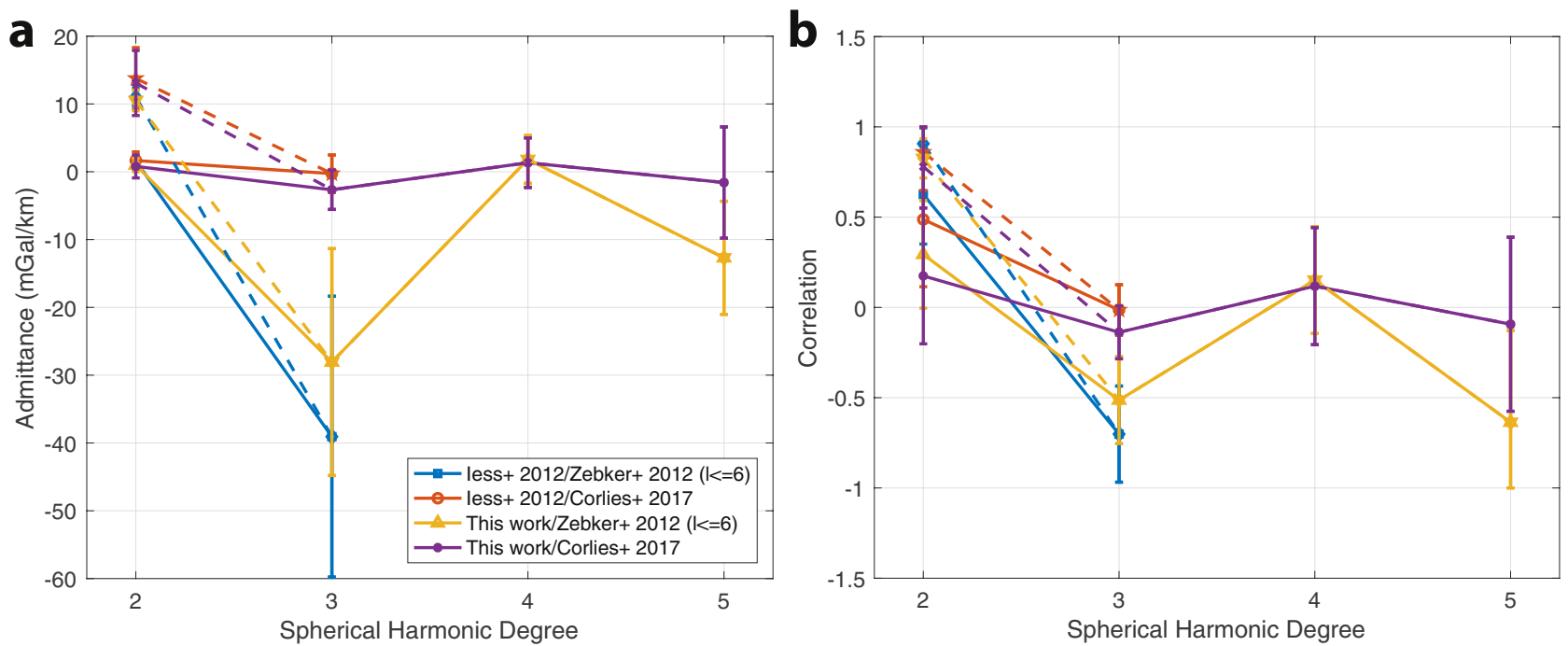

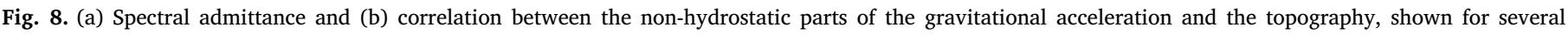

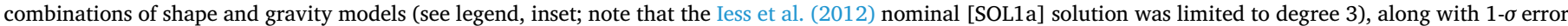

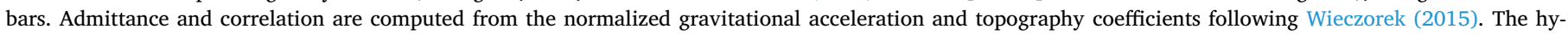
drostatic component is computed assuming a moment of inertia factor of either 0.341 (solid lines) or 0.32 (dashed lines).

thickening taking place due to melting/freezing at the base of the ice shell and if the ice shell is thick and stiff enough that elastic flexural support is important-though a rigid ice shell would also reduce the tidal $k_{2}$ Love number (see Hemingway et al., 2013). In fact, gravity/ topography correlation could even be negative if the ice shell is sufficiently stiff. An anti-correlation between Titan's degree-3 gravity and topography signals was previously noted by Hemingway et al. (2013) based on shape and gravity models available at the time (Iess et al., 2012; Zebker et al., 2012; blue lines in Fig. 8). When adopting the updated shape and gravity models, however, the correlation at degree 3 becomes significantly weaker (Figs. 7 and 8). Likewise, the correlations at degrees 4 and 5 are compatible with zero. Although strong compensation could help explain why the non-hydrostatic topography does not contribute much to the non-hydrostatic gravity, the appearance of uncorrelated non-hydrostatic gravity (Fig. 7, left column) suggests the presence of additional internal heterogeneities that have little or nothing to do with the topographic anomalies (Fig. 7, right column), perhaps pointing instead to anomalies within the rocky part of the interior (see also Palguta et al., 2006).

A possible exception to the lack of correlation occurs at degree 2, where the non-hydrostatic gravity and topography could be spatially correlated, depending on what is assumed for the moment of inertia factor. The assumption of hydrostatic equilibrium amounts to choosing the moment of inertia factor as the point on the hydrostatic line in Fig. 4a that is nearest to the measured gravity or, equivalently, to minimizing the non-hydrostatic part of the degree-2 gravity (i.e., minimizing the magnitude of the signal shown in Fig. 7a). Under this assumption, the non-hydrostatic degree- 2 gravity is negligible and so the degree-2 admittance is close to zero (Fig. 8a). Moments of inertia larger or smaller than 0.341 are, however, possible if we relax the assumption of hydrostatic equilibrium. As mentioned earlier, the measured $J_{2} / C_{22}$ ratio being close to $10 / 3$ is a necessary, but not sufficient condition for hydrostatic equilibrium. Indeed, at least one study favors a moment of inertia factor in the range $0.31-0.33$ in order to satisfy Titan's measured obliquity (Baland et al., 2014). In that scenario, the departure from hydrostatic equilibrium would involve more significant non-hydrostatic gravity, which would be positively correlated with the non-hydrostatic topography (i.e., both would involve excess power in the $l, m=2,0$ and $l, m=2,2$ terms). This is illustrated by the dashed lines showing the positive admittance (Fig. 8a) and correlation (Fig. 8b) computed from the degree- 2 non-hydrostatic gravity and topography when the moment of inertia factor is assumed to be 0.32 . This scenario does, however, require even the non-hydrostatic parts of $J_{2}$ and $C_{22}$ to be in a ratio close to $10 / 3$. Although such a ratio for the non-hydrostatic gravity terms would be fortuitous, the possibility cannot be excluded.

\section{Conclusion}

The analysis of ten Cassini flybys of Titan dedicated to gravity science proved that the acquired data have been adequate for a valuable determination of the gravity field of Saturn's largest moon. The gravity coefficients up to degree 5 have been reported, increasing the resolution of previous Cassini results, and providing insight into Titan's interior structure. The new solution confirms the previous result of Titan's large response to tidal forcing from Saturn: the confidence in the tidal Love number $k_{2}$ has been further increased, and the tidal time lag has been determined to be close to zero at 1- $\sigma$, indicating a phase angle lag smaller than $5.4^{\circ}$.

The new solution likewise confirms that, despite Titan's considerable excess flattening, its interior has relaxed to a state compatible with hydrostatic equilibrium with a moment of inertia factor close to 0.341 , though the presence of such significant non-hydrostatic topography is a reminder that hydrostatic equilibrium is not guaranteed, precluding a definitive determination of the moment of inertia. Comparison of the non-hydrostatic parts of the gravitational potential with the topography show that Titan's topography is highly compensated, as expected if the ice shell is thin (permitting effective compensation) or stiff (permitting overcompensation that remains effective even for a thicker ice shell). The weak spatial correlation between the non-hydrostatic gravity and topography beyond degree 2, however, may suggest the presence of internal mass anomalies at depth. A more strongly differentiated interior, with moment of inertia factor $<0.341$, is permitted but would imply less compensation at degree 2 .

Although new observational constraints on Titan's gravitational field must now await the next spacecraft mission to the Saturn system, increasingly comprehensive models, coupled with constraints from complementary observations (e.g., Titan's rotational state) and laboratory experiments, have the potential to yield further insights into Titan's interior and evolutionary history and to resolve many of the remaining puzzles. These include Titan's extreme excess flattening in spite of its apparently hydrostatic gravitational field, the nature of Titan's topography at various length scales and how it is maintained in spite of the 
basal ice being near its melting temperature (and therefore likely to undergo viscous relaxation), as well as the nature of those mass anomalies that are uncorrelated with the observed topography.

\section{Acknowledgments}

The work of D.D., P.R., and L.I. has been supported in part by the Italian Space Agency under ASI-INAF contract 2017-10-H.O. D.J.H. was supported in part by the Miller Institute for Basic Research in Science at the University of California, Berkeley.

\section{References}

Archinal, B.A., A'Hearn, M.F., Bowell, E., Conrad, A., Consolmagno, G.J., Courtin, R., Fukushima, T., Hestroffer, D., Hilton, J.L., Krasinsky, G.A., Neumann, G., Oberst, J., Seidelmann, P.K., Stooke, P., Tholen, D.J., Thomas, P.C., Williams, I.P., 2011. Report of the IAU working group on cartographic coordinates and rotational elements: 2009. Celestial Mechanics Dynamical Astronomy 109 (2), 101-135. https://doi.org/10. 1007/s10569-010-9320-4.

Archinal, B.A., Acton, C.H., A'Hearn, M.F., Conrad, A., Consolmagno, G.J., Duxbury, T., Hestroffer, D., Hilton, J.L., Kirk, R.L., Klioner, S.A., McCarthy, D., Meech, K., Oberst, J., Ping, J., Seidelmann, P.K., Tholen, D.J., Thomas, P.C., Williams, I.P., 2018. Report of the IAU working group on cartographic coordinates and rotational elements: 2015. Celestial Mechanics Dynamical Astronomy 130, 22. https://doi.org/10.1007/ s10569-017-9805-5.

Asmar, S.W., Armstrong, J.W., Iess, L., Tortora, P., 2005. Spacecraft Doppler tracking: noise budget and accuracy achievable in precision radio science investigations. Radio Sci. 40. https://doi.org/10.1029/2004RS003101.

Baland, R., Tobie, G., Lefèvre, A., Van Hoolst, T., 2014. Titan's internal structure inferred from its gravity field, shape, and rotation state. Icarus $237,29-41$. https://doi.org/ 10.1016/j.icarus.2014.04.007.

Barbaglio, F., Armstrong, J.W., Iess, L., 2012. Precise Doppler Measurements for Navigation and Planetary Geodesy Using Low Gain Antennas: Test Results from Cassini. (ISSFD 23rd).

Boone, D., J. Bellerose, and D. Roth (2017). Resolution of Orbit Determination Prediction Instabilities at Titan During Cassini's Solstice Mission. ISSFD-2017-109.

Choukron, M., Sotin, C., 2012. Is Titan's shape caused by its meteorology and carbon cycle? Geophys. Res. Lett. 39. https://doi.org/10.1029/2011GL050747.

Corlies, P., A. G. Hayes, S. P. D. Birch, R. Lorenz, B. W. Stiles, R. Kirk, V. Poggiali, H. Zebker, and L. Iess (2017). Titan's topography and shape at the end of the Cassini Mission. Geophys. Res. Lett., 44 (23), 11, 754-11, 761, doi:https://doi.org/10.1002/ 2017GL075518

Darwin, G.H., 1899. The theory of the figure of the earth carried to the second order in small quantities. Mon. Not. R. Astron. Soc. 60, 82-124. https://doi.org/10.1093/ mnras/60.2.82.

Ermakov, A.I., Park, R.S., Bills, B.G., 2018. Power laws of topography and gravity spectra of the solar system bodies. Journal of Geophysical Research: Planets 123, 2038-2064. https://doi.org/10.1029/2018JE005562.

Evans, S., Taber, W., Drain, T., Smith, J., Wu, H., Guevara, M., Sunseri, R., Evans, J., 2018. MONTE: the next generation of mission design and navigation software. CEAS Space Journal 10, 79-86. https://doi.org/10.1007/s12567-017-0171-7.

Fuller, J., Luan, J., Quataert, E., 2016. Resonance locking as the source of rapid tidal migration in the Jupiter and Saturn moon system. Mon. Not. R. Astron. Soc. 458, 3867-3879. https://doi.org/10.1093/mnras/stw609.

Hemingway, D.J., Nimmo, F., Zebker, H., Iess, L., 2013. A rigid and weathered ice shell on Titan. Nature 500 (7464), 550-552. https://doi.org/10.1038/nature12400.

Hemingway, D. J., L. Iess, R. Tajeddine, and G. Tobie (2018). "The interior of Enceladus," in Enceladus and the Icy Moons of Saturn, P. M. Schenk, R. N. Clark, C. J. A. Howett, and A. J. Verbiscer, Eds. Tucson, Arizona: University of Arizona Press, pp. 57-77.

Iess, L., Rappaport, N.J., Jacobson, R.A., Racioppa, P., Stevenson, D.J., Tortora, P., Armstrong, J.W., Asmar, S.W., 2010. Gravity field, shape, and moment of inertia of Titan. Science 327, 1367-1369. https://doi.org/10.1126/science.1182583.

Iess, L., Jacobson, R.A., Ducci, M., Stevenson, D.J., Lunine, J.I., Armstrong, J.W., Asmar, S.W., Racioppa, P., Rappaport, N.J., Tortora, P., 2012. The tides of Titan. Science 337, 457-459. https://doi.org/10.1126/science.1219631.

Iess, L., Di Benedetto, M., James, N., Mercolino, M., Simone, L., Tortora, P., 2014. Astra: interdisciplinary study on enhancement of the end-to-end accuracy of spacecraft tracking techniques. Acta Astronautica 94, 699-707. https://doi.org/10.1016/j. actaastro.2013.06.011.

Jacobson, R.A., Antreasian, P.G., Bordi, J.J., Criddle, K.E., Ionasescu, R., Jones, J.B., Mackenzie, R.A., Meek, M.C., Parcher, D., Pelletier, F.J., Owen, W.M., Roth, D.C., Roundhill, I.M., Stauch, J.R., 2006. The gravity field of the Saturnian system from satellite observations and spacecraft tracking data. Astron. J. 132, 2520-2526. https://doi.org/10.1086/508812.

Kaula, W.M., 1963. Determination of the Earth's gravitational field. Rev. Geophys. 1 (4), $507-551$.

Liu, H.S., Chao, B.F., 1991. The Earth's equatorial principal axes and moments of inertia Geophys. J. Int. 106, 699-702. https://doi.org/10.1111/j.1365-246X.1991. tb06341.x.

Matsuyama, I., 2011. Is Titan in Hydrostatic Equilibrium? In: EPSC-DPS2011-344-1.

Matsuyama, I., Beuthe, M., Hay, H.C.F.C., Nimmo, F., Kamata, S., 2018. Ocean tidal heating in icy satellites with solid shells. Icarus 312, 208-230. https://doi.org/10. 1016/j.icarus.2018.04.013.

Meriggiola, R., Iess, L., Stiles, B.W., Lunine, J.I., Mitri, G., 2016. The rotational dynamics of Titan from Cassini RADA images. Icarus 275, 183-192. https://doi.org/10.1016/j. icarus.2016.01.019.

Murray, C.D., Dermott, S.F., 1999. Solar System dynamics. Cambridge University Press0521-57295-9 ISBN.

NASA's Navigation and Ancillary Information Facility (NAIF) n.d. Saturn's satellite ephemerides can be found online: https://naif.jpl.nasa.gov/pub/naif/generic kernels/spk/satellites/

Nimmo, F., Bills, B.G., 2010. Shell thickness variations and the long-wavelength topography of titan. Icarus 208, 896-904. https://doi.org/10.1016/j.icarus.2010.02.020.

Palguta, J., Anderson, J.D., Shubert, G., Moore, W.B., 2006. Mass anomalies on Ganymede. Icarus 180, 428-441. https://doi.org/10.1016/j.icarus.2005.08.020.

Rappaport, N.J., Iess, L., Wahr, J., Lunine, J.I., Armstrong, J.W., Asmar, S.W., Tortora, P., Di Benedetto, M., Racioppa, P., 2008. Can Cassini detect a subsurface ocean in titan from gravity measurements? Icarus 194, 711-720. https://doi.org/10.1016/j.icarus. 2007.11.024.

Seidelmann, P.K., Abalakin, V.K., Bursa, M., Davies, M.E., De Bergh, C., Lieske, J.H., Simon, J.L., Standish, E.M., Stooke, P., Thomas, C., 2002. Report of the IAU/IAG Working Group on Cartographic Coordinates and Rotational Elements of the Planets and Satellites: 2000. Celestial Mechanics Dynamical. Astronomy 82, 83-110. https:// doi.org/10.1023/A:1013939327465.

Stiles, B.W., Kirk, R.L., Lorenz, R.D., Hensley, S., Lee, E., Ostro, S.J., Allison, M.D., Callahan, P.S., Gim, Y., Iess, L., Perci del marmot, P., Hamilton, G., Johnson, W.T.K., West, R.D., the Cassini RADAR team, 2008. Determining Titan's spin state from Cassini RADAR images. Astron. J. 135, 1669-1680. https://doi.org/10.1088/00046256/135/5/1669.

Tricarico, P., 2014. Multi-layer hydrostatic equilibrium of planets and synchronous moons: theory and application to Ceres and to solar system moons. Astrophys. J. 782, 99. https://doi.org/10.1088/0004-637X/782/2/992.

Wieczorek, M.A. (2015). "Gravity and topography of the terrestrial planets" in Treatise on Geophysics, 2nd edition, Elsevier. doi:https://doi.org/10.1016/B978-0-444-53802-4. 00169-X

Zebker, H.A., Stiles, B., Hensley, S., Lorenz, R., Kirk, R.L., Lunine, J., 2009. Size and shape of Saturn's moon Titan. Science 324 (5929), 921-923. https://doi.org/10.1126/ science.1168905.

Zebker, H.A., Iess, L., Wall, S.D., Lorenz, R.D., Lunine, J.I., Stiles, B.W., 2012. Titan's figure fatter, flatter than its gravity field. AGU Fall Meeting 2012, P23F-01. 